\title{
Galileo invariant system and the motion of relativistic $d$-branes*
}

\author{
D. Bazeia \\ Center for Theoretical Physics, Laboratory for Nuclear Science and Department of Physics \\ Massachusetts Institute of Technology, Cambridge, Massachusetts 02139-4307 \\ Departamento de Física, Universidade Federal da Paraíba \\ Caixa Postal 5008, 58051-970 João Pessoa, Paraíba, Brazil \\ MIT-CTP-2789
}

\begin{abstract}
We follow recent work and study the relativistic $d$-brane system in $(d+1,1)$ dimensions and its connection with a Galileo invariant system in $(d, 1)$ dimensions. In particular, we solve $d$-brane systems in $(2,1),(3,1)$ and $(4,1)$ dimensions and show that their solutions solve the corresponding Galileo invariant systems in $(1,1)$, $(2,1)$, and $(3,1)$ dimensions. The results are extended to higher dimensions.
\end{abstract}

PACS number(s): 11.27.+d, 03.40.Gc, 03.65.-w

\footnotetext{
${ }^{*}$ This work is supported in part by funds provided by the U.S. Department of Energy (D.O.E.) under cooperative research agreement \#DF-FC02-94ER40818, and by Conselho Nacional de Desenvolvimento Científico e Tecnológico, CNPq, Brazil
} 


\section{Introduction}

The main objective of this paper is to find solutions of the relativistic $d$-brane system in $(d+1,1)$ dimensions in connection with solutions of a Galileo invariant system in $(d, 1)$ dimensions, as recently considered in [1, 2]. Although we are mainly interested in the cases of $d=1,2$ and 3 spatial dimensions, some of the results will be naturally extended to $d>$ 1 , arbitrary. We remark that the Galileo invariant system is defined in $(d, 1)$ dimensions, and is related to the relativistic $d$-brane system in one higher spatial dimension, in $(d+1,1)$ dimensions. The relativistic $d$-brane is an extended object described by $\left(\phi^{0}, \phi^{1}, \ldots, \phi^{d}\right)$, where $\phi^{0}$ is the evolution parameter. The motion of the $d$-brane is governed by the Nambu-Goto action in $(d+1,1)$ space-time dimensions, with $x^{\mu}=\left(x^{0}, \mathbf{x}, x^{d+1}\right)$ and $\mathbf{x}=\left(x^{1}, \ldots, x^{d}\right)$. The Galileo invariant system was recently investigated in the work [1], [], and shows very interesting features, among them direct relation to fluid mechanics [3], to the hydrodynamical formulation of quantum mechanics [4, 5], and to relativistic membrane [6, 7] and its generalization to $d$-brane in $(d+1,1)$ dimensions. Other interesting issues are investigated in [8, 9], in [10] and also in [11, 12, 13].

The Galileo invariant system is a Lagrangian system governed by the pair of fields $\theta=\theta(t, \mathbf{x})$ and $\rho=\rho(t, \mathbf{x})$. Its dynamics is first-order in time, and the corresponding symplectic structure shows that $\theta(t, \mathbf{x})$ and $\rho(t, \mathbf{x})$ are canonically conjugate [14]. There are two equations of motion, one is a continuity equation for the density $\rho$ and the current $\mathbf{J}=\rho \nabla \theta$ and the other describes first-order time evolution of $\theta$, and depends on the presence of density-dependent interactions. The connection between such dynamical system and the membrane and its generalization to the $d$-brane system only appears under the very specific density-dependent interaction $V(\rho)=\lambda / \rho$.

In the work [1] some solutions of the dynamical system were presented in $(1,1)$ dimensions and in $(d, 1)$ for $d>1$. Also, in [2] the $(1,1)$ dimensional case was shown to be exactly solvable and solved explicitly. A lesson to be learned here is that this dynamical system seems to present very specific behavior in $(1,1)$ dimensions, differently of its general behavior in other dimensions $(d, 1)$ for $d>1$. This fact appears in the former investigations [1, 2] and also in the present investigations, which follows an alternate route to find solutions. We believe that the distinct behavior in $d=1$ is somehow related to the fact that the current density looses its vectorial character in one spatial dimension. In this work instead of dealing directly with the equations of motion that follows from the dynamical system we solve the $d$-brane system in $(d+1,1)$ dimensions. After solving the $d$-brane system we show how the solutions solve the corresponding Galileo invariant system. Although we shall be concerned mainly with the $d$-brane formulation of the problem, which is of direct interest to theoretical particle physics, we emphasize that the subject is of broader interest since it also offers connections to fluid dynamics [3], quantum mechanics [5], and other subjects [10, 11, 12, 13] of interest to physics.

The present work is organized as follows. In the next Sec. 2 we introduce the Galileo invariant system in $(d, 1)$ dimensions and the relativistic general $d$-brane system in $(d+1,1)$ 
dimensions, and we show the interesting connection between these two apparently distinct systems. We deal with specific issues in Sec. 3, where we split the subject into finding solutions of $d$-brane systems in $(4,1),(3,1)$ and in $(2,1)$ dimensions, and showing how they solve the corresponding Galileo invariant system in $(3,1),(2,1)$, and $(1,1)$ dimensions. In Sec. 1 we generalize results obtained in $d=1,2,3$ to $d>1$, arbitrary. Comments and conclusions are briefly presented in Sec. 0 .

\section{General considerations}

Here we introduce the Galileo invariant system and the relativistic $d$-brane system in $(d, 1)$ and in $(d+1,1)$ dimensions, respectively. The informations collected below follow in accordance with Refs. [1, 2], and are important for the issues that compose the rest of the paper. For simplicity we split the subject into the two subsections that follow.

\subsection{The Galileo invariant system in $(d, 1)$ dimensions}

The Galileo invariant system is governed by the action [1]

$$
I_{\lambda}=\int d t d \mathbf{x}\left(\theta \frac{\partial \rho}{\partial t}-\frac{1}{2} \rho \nabla \theta \cdot \nabla \theta-\frac{\lambda}{\rho}\right)
$$

Here we have $\mathbf{x}=\left(x^{1}, \ldots, x^{d}\right)$, and in this case we are working in $(d, 1)$ dimensions. This system is first-order in time [14] and the fields $\theta(t, \mathbf{x})$ and $\rho(t, \mathbf{x})$ are canonical variables, with the Poisson bracket

$$
\{\rho(t, \mathbf{x}), \theta(t, \mathbf{y})\}=\delta(\mathbf{x}-\mathbf{y})
$$

The Lagrangian and Hamiltonian are given by

$$
\begin{aligned}
L & =\int d \mathbf{x}\left(\theta \frac{\partial \rho}{\partial t}-\frac{1}{2} \rho \nabla \theta \cdot \nabla \theta-\frac{\lambda}{\rho}\right) \\
H & =\int d \mathbf{x}\left(\frac{1}{2} \rho \nabla \theta \cdot \nabla \theta+\frac{\lambda}{\rho}\right)
\end{aligned}
$$

There are two equations of motion. They can be obtained for instance via

$$
\begin{aligned}
& \frac{\partial \theta}{\partial t}=\{\theta, H\} \\
& \frac{\partial \rho}{\partial t}=\{\rho, H\}
\end{aligned}
$$


and they have the explicit form

$$
\begin{aligned}
& \frac{\partial \theta}{\partial t}+\frac{1}{2} \nabla \theta \cdot \nabla \theta=\frac{\lambda}{\rho^{2}} \\
& \frac{\partial \rho}{\partial t}+\nabla \cdot(\rho \nabla \theta)=0
\end{aligned}
$$

The first equation of motion couples $\theta$ to $\rho$ via the specific density-dependent interaction $V(\rho)=\lambda / \rho$. This equation decouples $\theta$ from $\rho$ in the absence of interactions, in the limit $\lambda \rightarrow 0$. The second equation, Eq. (8), is a continuity equation linking the density $\rho$ with the current density $\mathbf{J}=\rho \nabla \theta$. Here the current density necessarily couples the two fields $\rho$ and $\theta$. In one spatial dimension this coupling is non-vectorial, and we believe that this may be behind the very specific behavior this system presents in the $(1,1)$ dimensional case.

The hydrodynamical description of quantum mechanics follows with the Schrödinger action

$$
I_{S}=\int d t d \mathbf{x}\left(i \Psi^{*} \dot{\Psi}-\frac{1}{2} \nabla \Psi^{*} \cdot \nabla \Psi-V_{S}\right)
$$

where $V_{S}=V(t, \mathbf{x})+\bar{V}\left(\Psi^{*} \Psi\right)$ is the potential. We use

$$
\Psi(t, \mathbf{x})=\sqrt{\rho} e^{i \theta}
$$

and the specific potential

$$
V_{S}=\frac{\lambda}{\rho}-\frac{1}{8} \frac{(\nabla \rho)^{2}}{\rho}
$$

to make $I_{S}$ to collapse to $I_{\lambda}$, reproducing the two equations of motion (7) and (8). The quantum mechanical description of the system just introduced is then governed by the dynamical system with the action (1). This hydrodynamical description is restricted to pure states [15] and one may envisage another route, relying on the phase-space description of quantum mechanics [16], which seems appropriate to generalize the above description - recall that the (probability) density and the (probability) current density are the first two velocity moments of the Wigner distribution [17]. On the other hand, we remark that potentials like the one in Eq. (11) have been considered for instance in Ref. [18], and appear under the assumption of local probability conservation for the usual density and a current density that is extended to account for diffusion.

Although the dynamical system (11) is manifestaly invariant under Galileo transformations, it also presents [1] symmetry algebra that can be identified with that of the Poincaré group in $(d+1,1)$ dimensions. To see this, in $(d+1,1)$ dimensions we use light-cone coordinates to identify $\left(x^{0}, x^{1}, \ldots, x^{d+1}\right)$ with $(t, \theta, \mathbf{x})$, where $\mathbf{x}$ stands for the transverse coordinates and

$$
\begin{aligned}
t & \equiv \frac{1}{\sqrt{2}}\left(x^{0}+x^{d+1}\right)=x^{+} \\
\theta & \equiv \frac{1}{\sqrt{2}}\left(x^{0}-x^{d+1}\right)=x^{-}
\end{aligned}
$$


We now introduce the light-cone components of the Poincaré generators $P^{\mu}$ and $M^{\mu \nu}$ in the usual form

$$
\begin{aligned}
P^{\mu} & =\left(P^{-}, P^{+}, P^{i}\right) \\
M^{\mu \nu} & =\left(M^{+-}, M^{+i}, M^{-i}, M^{i j}\right)
\end{aligned}
$$

To identify the generators of the Poincaré symmetry we recall that the Poincaré group contains the extended Galileo group as a subgroup [19]. We write

$$
\begin{aligned}
P^{-} & \equiv H=\int d \mathbf{x} \mathcal{E} \\
P^{+} & \equiv N=\int d \mathbf{x} \rho \\
P^{i} & =\int d \mathbf{x} J^{i}
\end{aligned}
$$

and

$$
\begin{aligned}
M^{+-} & \equiv D=\int d \mathbf{x}(t \mathcal{E}-\theta \rho) \\
M^{+i} & \equiv B^{i}=\int d \mathbf{x}\left(t J^{i}-\rho x^{i}\right) \\
M^{-i} & \equiv G^{i}=\int d \mathbf{x}\left(\mathcal{E} x^{i}-\theta J^{i}\right) \\
M^{i j} & =\int d \mathbf{x}\left(x^{i} J^{j}-x^{j} J^{i}\right)
\end{aligned}
$$

The first set identifies generators of translations, which introduces the Hamiltonian $H$, charge $N$ and linear momentum $\mathbf{P}$. The second set identifies generators of rotations, which introduces the dilation $D$, Galileo boost $\mathbf{B}, \mathbf{G}$ and the angular momentum $\mathbf{M}$. Here we are using

$$
\begin{aligned}
J^{i} & =\rho \frac{\partial \theta}{\partial x^{i}} \\
\mathcal{E} & =\frac{1}{2} \rho \nabla \theta \cdot \nabla \theta+\frac{\lambda}{\rho}
\end{aligned}
$$

It is not hard to check that the generators (16)-(22) close the Poincaré algebra in $(d+1,1)$ dimensions.

It is interesting to realize that interchangeability of the light-cone coordinates $(+)$ and $(-)$ allows interchanging $t$ and $\theta$ in (12) and (13), and this further implies interchanging $P^{+}$and $P^{-}$and $M^{+i}$ and $M^{-i}$. We interchange $P^{+}$and $P^{-}$with $\rho \leftrightarrow \mathcal{E}$, which implies $H \leftrightarrow N$ and $\mathbf{B} \leftrightarrow-\mathbf{G}$. One verifies that under the interchange of light-cone coordinate the dilation changes as $D \leftrightarrow-D$, picking up the minus sign in the same way the other rotation generators $\mathbf{B}$ and $\mathbf{G}$ do. These properties make the identifications (16)-(17) and 
(19)-(21) very natural. Also they are important for constructing the explicit transformations: We offer a simple illustration by considering the generator of dilation symmetry; here we know that time changes according to

$$
t \rightarrow e^{w} t
$$

To make the interchangeability of light-cone coordinates to work correctly we must change the $\theta$ field according to

$$
\theta(t, \mathbf{x}) \rightarrow e^{w} \theta\left(e^{w} t, \mathbf{x}\right)
$$

which is indeed the correct transformation [1]. This reasoning is inspired on unpublished notes [20], and it helps building all the transformations explicitly, in particular the apparently misterious field-dependent coordinate transformations introduced in [1], which now follow naturally from the standard coordinate and field transformations that appear in a Galileo boost.

There is an alternate way to see the naturalness of the identification of the generators of the Lorentz symmetry. This was briefly introduced in [1], and here we make the argument explicit. We introduce the infinitesimal Lorentz transformations

$$
\delta x^{\mu}=w^{\mu \nu} x_{\nu}
$$

In the $(d+1,1)$ dimensional space-time we change to the former light-cone coordinates to rewrite the infinitesimal Lorentz transformations in the form

$$
\begin{aligned}
\delta x^{+} & =w^{+-} x^{+}+w^{i+} x^{i} \\
\delta x^{-} & =-w^{+-} x^{-}+w^{i-} x^{i} \\
\delta x^{i} & =w^{i-} x^{+}+w^{i+} x^{-}-w^{i j} x^{j}
\end{aligned}
$$

The parameters $w^{+-}, w^{i-}, w^{i+}$ and $w^{i j}$ respond for dilation, Galileo boost, field-dependent coordinate transformations and rotations, respectively. To see this explicitly we notice that the transformations involving only $w^{+-}$obey

$$
\begin{aligned}
& \delta x^{+}=w^{+-} x^{+} \\
& \delta x^{-}=-w^{+-} x^{-}
\end{aligned}
$$

We use $w^{+-}=w$ to see that $\delta^{(n)} x^{ \pm}=( \pm w)^{n} x^{ \pm}$. The finite transformations are

$$
\begin{aligned}
& \tilde{x}^{+}=e^{w} x^{+} \\
& \tilde{x}^{-}=e^{-w} x^{-}
\end{aligned}
$$

We use the light-cone identifications $x^{+}=t$ and $x^{-}=\theta_{w}(t, \mathbf{x})$ and write the new coordinates as $\tilde{x}^{+}=T$ and $\tilde{x}^{-}=\theta(T, \mathbf{X})$. We then get

$$
\begin{aligned}
t & \rightarrow T=e^{w} t \\
\theta & \rightarrow \theta_{w}(t, \mathbf{x})=e^{w} \theta\left(e^{w} t, \mathbf{x}\right)
\end{aligned}
$$


in agreement with the dilation transformations introduced in Ref. [1].

Let us now focus attention on the transformations introduced by $w^{i-}$, identifying $w^{i-}=w^{i}$. We obtain

$$
\begin{aligned}
\delta x^{+} & =0 \\
\delta x^{-} & =w^{i} x^{i} \\
\delta x^{i} & =w^{i} x^{+}
\end{aligned}
$$

We see that

$$
\begin{aligned}
\delta^{(2)} x^{+} & =0 \\
\delta^{(2)} x^{-} & =w^{2} x^{+} ; \quad \delta^{(3)} x^{-}=0 \\
\delta^{(2)} x^{i} & =0
\end{aligned}
$$

The finite transformations are then given by

$$
\begin{aligned}
T & =t \\
X^{i} & =x^{i}+w^{i} t
\end{aligned}
$$

and

$$
\theta(T, \mathbf{X})=\theta_{w}(t, \mathbf{x})+w^{i} x^{i}+\frac{1}{2} w^{2} t
$$

These transformations identify the Galileo boost - see Ref. [1].

We now examine the transformations introduced by $w^{i+}$, making the identification $w^{i+}=w^{i}$. We get

$$
\begin{aligned}
\delta x^{+} & =w^{i} x^{i} \\
\delta x^{-} & =0 \\
\delta x^{i} & =w^{i} x^{-}
\end{aligned}
$$

We see that

$$
\begin{aligned}
\delta^{(2)} x^{+} & =w^{2} x^{-} ; \quad \delta^{(3)} x^{+}=0 \\
\delta^{(2)} x^{-} & =0 \\
\delta^{(2)} x^{i} & =0
\end{aligned}
$$

The finite transformations are given by

$$
\begin{aligned}
T & =t+w^{i} x^{i}+\frac{1}{2} w^{2} \theta_{w}(t, \mathbf{x}) \\
X^{i} & =x^{i}+w^{i} \theta_{w}(t, \mathbf{x})
\end{aligned}
$$


and also

$$
\theta(T, \mathbf{X})=\theta_{w}(t, \mathbf{x})
$$

In this case we can write

$$
\begin{aligned}
T & =t+w^{i} x^{i}+\frac{1}{2} w^{2} \theta(T, \mathbf{X}) \\
X^{i} & =x^{i}+w^{i} \theta(T, \mathbf{X})
\end{aligned}
$$

which identify the field-dependent coordinate transformations introduced in Ref. [四].

The Galileo invariant system also engenders interesting solutions. The equations of motion (7) and (8) possess dilation-invariant solutions that can be written in the form

$$
\begin{aligned}
\theta(t, r) & =-\frac{r^{2}}{2(d-1) t} \\
\rho(t, r) & =\sqrt{\frac{2}{d} \lambda}(d-1) \frac{|t|}{r}
\end{aligned}
$$

This pair of solutions appears in $(d, 1)$ dimensions, for $d>1$, and here we have set $r=\sqrt{\mathbf{x} \cdot \mathbf{x}}$, which identifies the length of the vector $\mathbf{x}=\left(x^{1}, x^{2}, \ldots, x^{d}\right)$ in $d>1$ spatial dimensions. In Ref. [1] the pair of solutions for $d=2$ and the corresponding fielddependent coordinate transformations induced by the generators of $\mathbf{G}$ were used to obtain other solutions to the dynamical system in $(2,1)$ dimensions. Here, however, we explore other issues and get the pair of solutions (57) and (58) by following an alternate route, which relies on solving the $d$-brane problem in $(d+1,1)$ dimensions, for $d>1$.

This Galileo invariant system can also be solved in one spatial dimension, and some solutions were already presented in [1]. Also, in [2] it was shown that the $(1,1)$ dimensional system is integrable, and the explicit solutions were presented. This result is confirmed by the investigation that appears in Sec. 3 for the 1-brane system in $(2,1)$ dimensions.

\subsection{The $(d+1,1)$ dimensional $d$-brane system}

We follow Ref. [6] to introduce the relativistic $d$-brane system in $(d+1,1)$ dimensions. The $d$-brane system is an extended object described by the coordinates $\left(\phi^{0}, \phi^{1}, \ldots, \phi^{d}\right)$, where $\phi^{0}$ is the evolution parameter and $\left(\phi^{1}, \ldots, \phi^{d}\right)$ constitute the $d$-dimensional space that parametrizes the $d$-brane. This object is governed by the Nambu-Goto action

$$
I_{d}=-\int d \phi^{0} d \phi^{1} \ldots d \phi^{d} \sqrt{G}
$$

where $G$ is $(-1)^{d}$ times the determinant of the induced metric

$$
G_{\alpha \beta} \equiv \frac{\partial x^{\mu}}{\partial \phi^{\alpha}} \frac{\partial x_{\mu}}{\partial \phi^{\beta}}
$$


where $\alpha, \beta=0,1, \ldots, d$. Here the $d$-brane is submersed in the $(d+1,1)$ spacetime and we use light-cone coordinates to represent $x^{\mu}=\left(x^{0}, x^{1}, \ldots, x^{d}, x^{(d+1)}\right)$ as $(\tau, \theta, \mathbf{x})$, where

$$
\begin{aligned}
& x^{+}=\frac{1}{\sqrt{2}}\left(x^{0}+x^{(d+1)}\right) \equiv \tau \\
& x^{-}=\frac{1}{\sqrt{2}}\left(x^{0}-x^{(d+1)}\right) \equiv \theta
\end{aligned}
$$

We use light-cone coordinates with the same motivation of the former case, where we have shown that the Galileo invariant system in $(d, 1)$ dimensions presents symmetry algebra that can be identified with that of the Poincaré group in $(d+1,1)$ dimensions. This is convenient because light-cone coordinates introduce the tranverse spatial components $\mathbf{x}=\left(x^{1}, \ldots, x^{d}\right)$ very naturally, which we shall identify directly with the spatial components of the Galileo invariant system in $(d, 1)$ dimensions.

We identify the evolution parameter $\phi^{0}$ with the light-cone time $\tau\left(\tau \equiv \phi^{0}\right)$ to write the elements of $G_{\alpha \beta}$ in the form

$$
\begin{aligned}
G_{00} & =2 \frac{\partial \theta}{\partial \tau}-\frac{\partial \mathbf{x}}{\partial \tau} \cdot \frac{\partial \mathbf{x}}{\partial \tau} \\
G_{0 i} & =G_{i 0}=\frac{\partial \theta}{\partial \phi^{i}}-\frac{\partial \mathbf{x}}{\partial \tau} \cdot \frac{\partial \mathbf{x}}{\partial \phi^{i}} \\
G_{i j} & =-g_{i j}=\frac{\partial \mathbf{x}}{\partial \phi^{i}} \cdot \frac{\partial \mathbf{x}}{\partial \phi^{j}}
\end{aligned}
$$

We write

$$
g \equiv \operatorname{det}\left(g_{i j}\right)
$$

to get

$$
G=g\left(2 \frac{\partial \theta}{\partial \tau}-\frac{\partial \mathbf{x}}{\partial \tau} \cdot \frac{\partial \mathbf{x}}{\partial \tau}+g^{i j} u_{i} u_{j}\right)
$$

where $g^{i k} g_{k j}=\delta_{j}^{i}$ and $u_{i}$ is defined as

$$
u_{i} \equiv-\frac{\partial \theta}{\partial \phi^{i}}+\frac{\partial \mathbf{x}}{\partial \tau} \cdot \frac{\partial \mathbf{x}}{\partial \phi^{i}}
$$

The equations of motion for the $d$-brane can be written in the form [6]

$$
\begin{aligned}
\frac{\partial \mathbf{x}}{\partial \tau} & =-\frac{\mathbf{p}}{\Pi}+u^{i} \frac{\partial \mathbf{x}}{\partial \phi^{i}} \\
\frac{\partial \theta}{\partial \tau} & =\frac{1}{2 \Pi^{2}}(\mathbf{p} \cdot \mathbf{p}+g)+u^{i} \frac{\partial \theta}{\partial \phi^{i}} \\
\frac{\partial \mathbf{p}}{\partial \tau} & =-\frac{\partial}{\partial \phi^{i}}\left(\frac{1}{\Pi} g g^{i j} \frac{\partial \mathbf{x}}{\partial \phi^{j}}\right)+\frac{\partial}{\partial \phi^{i}}\left(\mathbf{p} u^{i}\right) \\
\frac{\partial \Pi}{\partial \tau} & =\frac{\partial}{\partial \phi^{i}}\left(\Pi u^{i}\right)
\end{aligned}
$$


where $\mathbf{p}$ and $\Pi$ are canonical momenta conjugate to $\mathbf{x}$ and $\theta$, respectively. The motion is constrained to obey

$$
\mathbf{p} \cdot \frac{\partial \mathbf{x}}{\partial \phi^{i}}+\Pi \frac{\partial \theta}{\partial \phi^{i}}=0
$$

This condition appears as a consequence of gauge symmetry, which comes from the freedom to parametrize the $d$-brane.

In the light-cone coordinates with the time identification $\tau \equiv \phi^{0}$ we can further investigate the physical contents of the $d$-brane after seting $u_{i}=0$. This condition identifies the (light-cone) time dependence of the parametrization and allows rewriting the equations of motion in the simpler form

$$
\begin{aligned}
\mathbf{p} & =-\Pi \frac{\partial \mathbf{x}}{\partial \tau} \\
\frac{\partial \Pi}{\partial \tau} & =0
\end{aligned}
$$

and

$$
\begin{aligned}
\frac{\partial \theta}{\partial \tau} & =\frac{1}{2}\left(\frac{\partial \mathbf{x}}{\partial \tau} \cdot \frac{\partial \mathbf{x}}{\partial \tau}+\frac{1}{\Pi^{2}} g\right) \\
\frac{\partial^{2} \mathbf{x}}{\partial \tau^{2}} & =\frac{1}{\Pi} \frac{\partial}{\partial \phi^{i}}\left(\frac{1}{\Pi} g g^{i j} \frac{\partial \mathbf{x}}{\partial \phi^{j}}\right)
\end{aligned}
$$

Instead of Eq. (73) the constrained motion now obeys

$$
\frac{\partial \theta}{\partial \phi^{i}}=\frac{\partial \mathbf{x}}{\partial \tau} \cdot \frac{\partial \mathbf{x}}{\partial \phi^{i}}
$$

which reproduces Eq. (68) for $u_{i}=0$ in a self-consistent way. The constraint Eq. (78) is independent of the explicit form of the momentum $\Pi=\Pi\left(\phi^{1}, \ldots, \phi^{d}\right)$, now $\tau$-independent in accordance with Eq. (75). This fact shows that one is still free to make $\tau$-independent reparametrization $\left(\phi^{1}, \ldots, \phi^{d}\right) \rightarrow\left(\bar{\phi}^{1}, \ldots, \bar{\phi}^{d}\right)$ of the $d$-brane. Different choices of the momentum $\Pi$ are related to different choices of $\tau$-independent parametrization and then we can choose $\Pi$ at convenience. For instance, the choice $\Pi=-c w\left(\phi^{1}, \ldots, \phi^{d}\right)$ envolving a constant $c$ times a specified function of $\phi^{1}, \ldots, \phi^{d}$ is known [6] as the orthonormal gauge. We can illustrate this point by going from $\Pi\left(\phi^{1}, \ldots, \phi^{d}\right)$ to $\Pi=-c$, constant, for simplicity. In this case the above Eqs. (76) and (77) become

$$
\begin{aligned}
\frac{\partial^{2} \mathbf{x}}{\partial \tau^{2}} & =\frac{\partial}{\partial \phi^{i}}\left(\frac{1}{c^{2}} g g^{i j} \frac{\partial \mathbf{x}}{\partial \phi^{j}}\right) \\
\frac{\partial \theta}{\partial \tau} & =\frac{1}{2}\left(\frac{\partial \mathbf{x}}{\partial \tau} \cdot \frac{\partial \mathbf{x}}{\partial \tau}+\frac{1}{c^{2}} g\right)
\end{aligned}
$$


We now change parametrization by allowing

$$
\frac{\partial \mathbf{x}}{\partial \phi^{i}} \rightarrow \frac{\partial \mathbf{x}}{\partial \bar{\phi}^{j}} \frac{\partial \bar{\phi}^{j}}{\partial \phi^{i}}
$$

with a similar change in $\theta\left(\tau, \phi^{1}, \ldots, \phi^{d}\right)$, in accordance with the constraint Eq. (78). We use

$$
\frac{\partial \bar{\phi}^{i}}{\partial \phi^{j}}=c \delta_{j}^{i}
$$

as the choice to get rid of the factor $1 / c^{2}$ in Eqs. (79) and (80). For simplicity we then consider $\Pi=-1$, which further implies $\mathbf{p}=\partial \mathbf{x} / \partial \tau$. In this case the equations of motion simplify to the equations

$$
\begin{aligned}
\frac{\partial^{2} \mathbf{x}}{\partial \tau^{2}} & =\frac{\partial}{\partial \phi^{i}}\left(g g^{i j} \frac{\partial \mathbf{x}}{\partial \phi^{j}}\right) \\
\frac{\partial \theta}{\partial \tau} & =\frac{1}{2}\left(\frac{\partial \mathbf{x}}{\partial \tau} \cdot \frac{\partial \mathbf{x}}{\partial \tau}+g\right)
\end{aligned}
$$

The constraint Eq. (78) was first solved [7] in the $d=2$ case, the membrane case and it can also be solved in the general $d$-brane case. We follow Ref. [2] and here the main step concerns inverting $\mathbf{x}=\mathbf{x}\left(\tau, \phi^{1}, \ldots, \phi^{d}\right)$ to get $\phi^{i}=\phi^{i}(t, \mathbf{x})$, after renaming $\tau$ as time $t$. Here we have

$$
\frac{\partial}{\partial \tau}=\frac{\partial}{\partial t}+\nabla \theta \cdot \nabla
$$

and the constraint Eq. (78) is solved by

$$
\frac{\partial \mathbf{x}}{\partial \tau}=\nabla \theta
$$

In this case Eq. (84) becomes

$$
\frac{\partial \theta}{\partial t}+\frac{1}{2} \nabla \theta \cdot \nabla \theta=\frac{1}{2} g
$$

and reproduces Eq. (7) if and only if we further define

$$
g \equiv \frac{2 \lambda}{\rho^{2}}
$$

On the other hand the Eq. (83) can be rewritten as

$$
\frac{\partial^{2} x^{i}}{\partial \tau^{2}}=\frac{1}{2} \frac{\partial g}{\partial x^{i}}
$$


The proof follows after recognizing that

$$
g g^{i j}=\frac{1}{(d-1) !} \epsilon^{i i_{2} \ldots i_{d}} \epsilon^{j j_{2} \ldots j_{d}} \frac{\partial x^{k_{2}}}{\partial \phi^{i_{2}}} \cdots \frac{\partial x^{k_{d}}}{\partial \phi^{i_{d}}} \frac{\partial x^{k_{2}}}{\partial \phi^{j_{2}}} \cdots \frac{\partial x^{k_{d}}}{\partial \phi^{j_{d}}}
$$

In this case we can use

$$
\epsilon^{j j_{2} \ldots j_{d}} \frac{\partial x^{k_{2}}}{\partial \phi^{j_{2}}} \cdots \frac{\partial x^{k_{d}}}{\partial \phi^{j_{d}}} \frac{\partial x^{k}}{\partial \phi^{j}} \equiv \epsilon^{k k_{2} \ldots k_{d}} \sqrt{g}
$$

to get to

$$
g g^{i j} \frac{\partial x^{k}}{\partial \phi^{j}}=\frac{1}{(d-1) !} \epsilon^{i i_{2} \ldots i_{d}} \epsilon^{k k_{2} \ldots k_{d}} \frac{\partial x^{k_{2}}}{\partial \phi^{i_{2}}} \cdots \frac{\partial x^{k_{d}}}{\partial \phi^{i_{d}}} \sqrt{g}
$$

and now we can write, recalling the smoothness of $\mathbf{x}=\mathbf{x}\left(\tau, \phi^{1}, \ldots, \phi^{d}\right)$ and (anti) symmetry of the Levi-Civita symbol,

$$
\begin{aligned}
\frac{\partial}{\partial \phi^{i}}\left(g g^{i j} \frac{\partial x^{k}}{\partial \phi^{j}}\right) & =\frac{1}{(d-1) !} \epsilon^{i i_{2} \ldots i_{d}} \epsilon^{k k_{2} \ldots k_{d}} \frac{\partial x^{k_{2}}}{\partial \phi^{i_{2}}} \cdots \frac{\partial x^{k_{d}}}{\partial \phi^{i_{d}}}\left[\frac{\partial}{\partial \phi^{i}}(\sqrt{g})\right] \\
& =\frac{1}{(d-1) !} \epsilon^{i i_{2} \ldots i_{d}} \epsilon^{k k_{2} \ldots k_{d}} \frac{\partial x^{k_{2}}}{\partial \phi^{i_{2}}} \cdots \frac{\partial x^{k_{d}}}{\partial \phi^{i_{d}}}\left[\frac{\partial}{\partial x^{l}}(\sqrt{g})\right] \frac{\partial x^{l}}{\partial \phi^{i}} \\
& =\frac{1}{(d-1) !} \epsilon^{l k_{2} \ldots k_{d}} \epsilon^{k k_{2} \ldots k_{d}} \sqrt{g}\left[\frac{\partial}{\partial x^{l}}(\sqrt{g})\right]
\end{aligned}
$$

We use the identity

$$
\frac{1}{(d-1) !} \epsilon^{i k_{2} \ldots k_{d}} \epsilon^{j k_{2} \ldots k_{d}} \equiv \delta^{i j}
$$

to obtain

$$
\frac{\partial}{\partial \phi^{i}}\left(g g^{i j} \frac{\partial x^{k}}{\partial \phi^{j}}\right)=\frac{1}{2} \frac{\partial g}{\partial x^{k}}
$$

which ends the proof. We then use Eqs. (85) and (86) to write

$$
\begin{aligned}
\frac{\partial^{2} \mathbf{x}}{\partial \tau^{2}} & =\frac{\partial}{\partial \tau} \nabla \theta \\
& =\nabla \frac{\partial \theta}{\partial t}+\nabla \theta \cdot \nabla(\nabla \theta) \\
& =\nabla\left(\frac{\partial \theta}{\partial t}+\frac{1}{2} \nabla \theta \cdot \nabla \theta\right)
\end{aligned}
$$

We use this together with Eq. (89) to obtain, discarding an unimportant constant,

$$
\frac{\partial \theta}{\partial t}+\frac{1}{2} \nabla \theta \cdot \nabla \theta=\frac{1}{2} g
$$


This equation also reproduces the Eq. (17) for $g$ defined by Eq. (88).

The Galileo invariant system (11) contains another equation of motion. It is Eq. (8), the continuity equation. It is obtained from the relativistic $d$-brane system as follows. We have, by definition

$$
\frac{\partial g}{\partial \tau} \equiv g g^{i j} \frac{\partial}{\partial \tau} g_{i j}
$$

Also

$$
\frac{\partial g}{\partial \tau}=\frac{\partial g}{\partial t}+\nabla \theta \cdot \nabla g
$$

We use these results together with

$$
\begin{aligned}
g^{i j} \frac{\partial}{\partial \tau} g_{i j} & =\frac{\partial \phi^{i}}{\partial x^{k}} \frac{\partial \phi^{j}}{\partial x^{k}} \frac{\partial}{\partial \tau}\left(\frac{\partial x^{l}}{\partial \phi^{i}} \frac{\partial x^{l}}{\partial \phi^{j}}\right) \\
& =2 \frac{\partial \phi^{j}}{\partial x^{k}} \frac{\partial}{\partial \phi^{j}} \frac{\partial x^{k}}{\partial \tau} \\
& =2 \frac{\partial}{\partial x^{k}} \frac{\partial \theta}{\partial x^{k}}
\end{aligned}
$$

to obtain

$$
\frac{\partial g}{\partial t}+\nabla \theta \cdot \nabla g=2 g \nabla^{2} \theta
$$

which reproduces the continuity equation after choosing $g=2 \lambda / \rho^{2}$, as given by Eq. (88). The motion of the $d$-brane in $(d+1,1)$ dimensions is then governed by the equations of motion that describe the dynamical system (1) in $(d, 1)$ dimensions. We remark that the above proof only works under the definition introduced in Eq. (88), with the densitydependent interaction described by the very specific potential $V(\rho)=\lambda / \rho$.

There is an alternate way to make the $d$-brane system in $(d+1,1)$ dimensions to reproduce the Galileo invariant system in $(d, 1)$ dimensions. It follows like in Ref. [8], for instance, but now in $d+1$ spatial dimensions. It relies on reformulating the description of the $d$-brane according to the identifications 21

$$
\begin{aligned}
& x^{+}=\frac{1}{\sqrt{2}}\left(x^{0}+x^{d+1}\right) \equiv t=\phi^{0} \\
& x^{-}=\frac{1}{\sqrt{2}}\left(x^{0}-x^{d+1}\right) \equiv \theta
\end{aligned}
$$

and also

$$
\phi^{i} \equiv x^{i}, \quad i=1,2, \ldots, d
$$

This is interesting since now $\theta=\theta(t, \mathbf{x})$ is directly identified with the $\theta$ field of the Galileo invariant system in $(d, 1)$ dimensions. To see this explictly we recall from Eq. (59) that the Lagrangian density can be written as

$$
\mathcal{L}_{d}=\sqrt{G}
$$


and now

$$
G=2 \frac{\partial \theta}{\partial t}+\nabla \theta \cdot \nabla \theta
$$

The momentum conjugate to $\theta$ is given by

$$
\Pi=\frac{1}{\sqrt{2 \frac{\partial \theta}{\partial t}+\nabla \theta \cdot \nabla \theta}}
$$

and this gives the Hamiltonian density

$$
\mathcal{H}=-\frac{1}{2 \Pi}-\frac{1}{2} \Pi \nabla \theta \cdot \nabla \theta
$$

We use this Hamiltonian density to write the first-order Lagrangian density, after defining $\Pi \equiv-\rho / \sqrt{2 \lambda}$. We discard a total time derivative and ignore an unimportant multiplicative constant to obtain

$$
\mathcal{L}=\theta \frac{\partial \rho}{\partial t}-\frac{1}{2} \rho \nabla \theta \cdot \nabla \theta-\frac{\lambda}{\rho}
$$

This is exactly the Lagrangian density that follows from the action (11) that defines the Galileo invariant system in $(d, 1)$ dimensions.

\section{Some brane solutions}

Let us now deal with solutions of $d$-brane systems in $(2,1),(3,1)$ and $(4,1)$ dimensions, and with the relations between such solutions and solutions of the Galileo invariant system in one, two and three spatial dimensions. We split the subject into the three subsections that follow, which deal with $d=1,2,3$ separately.

\subsection{Solutions for $d=1$}

Here we consider the relativistic 1-brane system in $(2,1)$ dimensions. We parametrize the system with $\phi$, and the unidimensional transverse coordinate is given by $x=x(\tau, \phi)$. Also, the matrix $g_{i j}=\left(\partial \mathbf{x} / \partial \phi^{i}\right) \cdot\left(\partial \mathbf{x} / \partial \phi^{j}\right)$ and its determinant $g$ now become the very same thing, explicitly

$$
g=\left(\frac{\partial x}{\partial \phi}\right)^{2}
$$

Also, $g g^{i j} \rightarrow 1$ and the equation of motion (83) becomes

$$
\frac{\partial^{2} x}{\partial \tau^{2}}-\frac{\partial^{2} x}{\partial \phi^{2}}=0
$$


The other Eqs. (78) and (84) give

$$
\begin{aligned}
& \frac{\partial \theta}{\partial \phi}=\frac{\partial x}{\partial \tau} \frac{\partial x}{\partial \phi} \\
& \frac{\partial \theta}{\partial \tau}=\frac{1}{2}\left[\left(\frac{\partial x}{\partial \tau}\right)^{2}+\left(\frac{\partial x}{\partial \phi}\right)^{2}\right]
\end{aligned}
$$

We can solve Eq. (111) directly. It is a wave equation and presents the general solution

$$
x(\tau, \phi)=f_{+}(\tau+\phi)+f_{-}(\tau-\phi)
$$

The solution is written in terms of two arbitrary functions $f_{ \pm}$, and here we have

$$
\frac{\partial x}{\partial \tau}=f_{+}^{\prime}(\tau+\phi)+f_{-}^{\prime}(\tau-\phi)
$$

and

$$
\frac{\partial x}{\partial \phi}=f_{+}^{\prime}(\tau+\phi)-f_{-}^{\prime}(\tau-\phi)
$$

where we are using the notation $f^{\prime}(z)=(\partial f / \partial z)$. We use these expressions to rewrite Eqs. (112) and (113) as

$$
\begin{aligned}
& \frac{\partial \theta}{\partial \tau}=\left[f_{+}^{\prime}(\tau+\phi)\right]^{2}+\left[f_{-}^{\prime}(\tau-\phi)\right]^{2} \\
& \frac{\partial \theta}{\partial \phi}=\left[f_{+}^{\prime}(\tau+\phi)\right]^{2}-\left[f_{-}^{\prime}(\tau-\phi)\right]^{2}
\end{aligned}
$$

in order to get

$$
\begin{aligned}
& \frac{1}{2}\left(\frac{\partial}{\partial \tau}+\frac{\partial}{\partial \phi}\right) \theta(\tau, \phi)=\left[f_{+}^{\prime}(\tau+\phi)\right]^{2} \\
& \frac{1}{2}\left(\frac{\partial}{\partial \tau}-\frac{\partial}{\partial \phi}\right) \theta(\tau, \phi)=\left[f_{-}^{\prime}(\tau-\phi)\right]^{2}
\end{aligned}
$$

These results allow writing $\theta(\tau, \phi)=\theta_{+}(\tau+\phi)+\theta_{-}(\tau-\phi)$, and the general solution for $\theta$ can be written in the form

$$
\theta(\tau, \phi)=\int^{(\tau+\phi)}\left[f_{+}^{\prime}(z)\right]^{2} d z+\int^{(\tau-\phi)}\left[f_{-}^{\prime}(z)\right]^{2} d z
$$

The results given by Eqs. (114) and (121) constitute the pair of general solutions $\mathbf{x}(\tau, \phi)$ and $\theta(\tau, \phi)$ of the 1-brane system.

To connect the 1-brane system to the Galileo invariant system in $(1,1)$ dimensions we follow the general investigations introduced in Sec. 2. We start by rewriting $\theta(\tau, \phi)$ as 
$\theta(t, x)$. Since we already have $x=x(\tau, \phi)$ in Eq. (114), we write $\phi=h(\tau, x)$ and change $\tau \rightarrow t$ to get to

$$
x \equiv f_{+}(t+h(t, x))+f_{-}(t-h(t, x))
$$

in a way such that

$$
\left(1+h_{t}\right) f_{+}^{\prime}+\left(1-h_{t}\right) f_{-}^{\prime}=0
$$

and

$$
h_{x} f_{+}^{\prime}-h_{x} f_{-}^{\prime}=1
$$

We also get

$$
h_{t}=\frac{\partial h}{\partial t}=-\frac{f_{+}^{\prime}+f_{-}^{\prime}}{f_{+}^{\prime}-f_{-}^{\prime}}
$$

and

$$
h_{x}=\frac{\partial h}{\partial x}=\frac{1}{f_{+}^{\prime}-f_{-}^{\prime}}
$$

These results allow writing

$$
\theta(t, x)=\int^{[t+h(x, t)]}\left[f_{+}^{\prime}(z)\right]^{2} d z+\int^{[t-h(x, t)]}\left[f_{-}^{\prime}(z)\right]^{2} d z
$$

On the other hand, we use Eq. (88) to get

$$
g=\left(\frac{\partial x}{\partial \phi}\right)^{2}=\frac{2 \lambda}{\rho^{2}}
$$

and this leads to the result

$$
\rho(t, x)= \pm \frac{\sqrt{2 \lambda}}{f_{+}^{\prime}-f_{-}^{\prime}}
$$

The above field configurations $\theta(t, x)$ and $\rho(t, x)$ obey the pair of equations

$$
\begin{aligned}
\frac{\partial \rho}{\partial t}+\frac{\partial}{\partial x}\left[\rho\left(\frac{\partial \theta}{\partial x}\right)\right] & =0 \\
\frac{\partial \theta}{\partial t}+\frac{1}{2}\left(\frac{\partial \theta}{\partial x}\right)^{2} & =\frac{\lambda}{\rho^{2}}
\end{aligned}
$$

which are the equations of motion (7) and (8) in the $(1,1)$ dimensional case.

Furthermore, we can use Eq. (127) to write

$$
\frac{\partial \theta}{\partial t}=-2 f_{+}^{\prime} f_{-}^{\prime}
$$

Also, the current density $J(x, t)=\rho(\partial \theta / \partial x)$ can be written as

$$
J(t, x)= \pm \sqrt{2 \lambda} \frac{f_{+}^{\prime}+f_{-}^{\prime}}{f_{+}^{\prime}-f_{-}^{\prime}}
$$


It is now interesting to see that if we set $f_{+}$or $f_{-}$to zero, that is, if we solve Eq. (111) with only one of the two independent solutions we get to the result that both $\theta$ and $\rho$ are time-independent: We see that $\theta$ is time-independent directly from Eq. (132); from Eq. (133) we get that the current density is constant, and so the continuity equation demands the density to be time-independent. We remark that the equations of motion (130) and (131) impose that the current density is a specific constant

$$
J(t, x)= \pm \sqrt{2 \lambda}
$$

when one considers time-independent configurations. This means that the particular solution $x(\tau, \phi)=f_{+}(\tau+\phi)$ or $x(\tau, \phi)=f_{-}(\tau-\phi)$ to the Eq. (111) reproduces all the static solutions of the dynamical system governed by the action (1) in $(1,1)$ dimensions

For the $(1,1)$ dimensional system the energy of static configurations $\theta=\theta(x)$ and $\rho=\rho(x)$ can be written in the form

$$
\begin{aligned}
E & =\int d x\left[\frac{1}{2} \rho\left(\frac{d \theta}{d x}\right)^{2}+\frac{\lambda}{\rho}\right] \\
& = \pm \sqrt{2 \lambda} \int d x \frac{d \theta}{d x}+\frac{1}{2} \int d x \rho\left(\frac{d \theta}{d x} \mp \frac{\sqrt{2 \lambda}}{\rho}\right)^{2}
\end{aligned}
$$

and so is minimized to the value

$$
E_{M}= \pm \sqrt{2 \lambda}[\theta(x=\infty)-\theta(x=-\infty)]
$$

for field configurations that obey

$$
\frac{d \theta}{d x}= \pm \frac{\sqrt{2 \lambda}}{\rho}
$$

which are the same solutions with constant and uniform current density already obtained. Here we notice that the energy can also be written as

$$
E_{M}=\int d x \rho\left(\frac{d \theta}{d x}\right)^{2}=2 \int d x \frac{\lambda}{\rho}
$$

This shows that the kinetic and potential parts of the energy contritube equally, as usually happens with BPS solutions [22]. This means that the system described by the Eqs. (130) and (131) is the bosonic portion of some supersymmetric system, and that the 1-brane solutions with $(\tau+\phi)$ or $(\tau-\phi)$ are the solutions that generate all the BPS solutions of the corresponding dynamical system described by the action (11) in $(1,1)$ dimensions.

\subsection{Solutions for $d=2$}

Let us consider the $(3,1)$ dimensional problem. This is the case with $d=2$, the membrane case where $\left(\phi^{1}, \phi^{2}\right)=(\phi, \psi)$ and $\mathbf{x}=(x, y)$. This problem was already considered in 
[6. 17, 8]. Here we have

$$
\begin{aligned}
& g_{11}=\left(\frac{\partial x}{\partial \phi}\right)^{2}+\left(\frac{\partial y}{\partial \phi}\right)^{2} \\
& g_{12}=g_{21}=\frac{\partial x}{\partial \phi} \frac{\partial x}{\partial \psi}+\frac{\partial y}{\partial \phi} \frac{\partial y}{\partial \psi} \\
& g_{22}=\left(\frac{\partial x}{\partial \psi}\right)^{2}+\left(\frac{\partial y}{\partial \psi}\right)^{2}
\end{aligned}
$$

and we get

$$
g=\left(\frac{\partial x}{\partial \phi} \frac{\partial y}{\partial \psi}-\frac{\partial x}{\partial \psi} \frac{\partial y}{\partial \phi}\right)^{2}
$$

We can write $g=\{x, y\}^{2}$, where

$$
\{x, y\}=\frac{\partial x}{\partial \phi} \frac{\partial y}{\partial \psi}-\frac{\partial x}{\partial \psi} \frac{\partial y}{\partial \phi}
$$

is the Poisson bracket with respect to the membrane coordinates $(\phi, \psi)$. We remark that such identification is only possible in the membrane case, for $d=2$.

In Ref. [G] one finds solutions of the Galileo invariant system in the planar $(d=2)$ case, which present dilation and circular symmetries. For this reason we choose the circular Ansatz

$$
\begin{aligned}
& x=R(\tau, \phi) \cos (\psi) \\
& y=R(\tau, \phi) \sin (\psi)
\end{aligned}
$$

In this case we get

$$
\begin{aligned}
& g_{11}=\left(\frac{\partial R}{\partial \phi}\right)^{2} \\
& g_{12}=g_{21}=0 \\
& g_{22}=R^{2}
\end{aligned}
$$

which gives

$$
g=R^{2}\left(\frac{\partial R}{\partial \phi}\right)^{2}
$$

We also have

$$
\begin{aligned}
& g g^{11}=R^{2} \\
& g g^{12}=g g^{21}=0 \\
& g g^{22}=\left(\frac{\partial R}{\partial \phi}\right)^{2}
\end{aligned}
$$


We use the equation of motion (83) to get from the equations for $x$ and $y$ the same equation for $R=R(\tau, \phi)$

$$
\frac{\partial^{2} R}{\partial \tau^{2}}=R^{2} \frac{\partial^{2} R}{\partial \phi^{2}}+R\left(\frac{\partial R}{\partial \phi}\right)^{2}
$$

This equation is solved by separating variables. Here we get

$$
R(\tau, \phi)=2^{1 / 2} \frac{\phi}{\tau}
$$

The other Eqs. (88) and (84) give

$$
\theta(\tau, \phi)=-\frac{\phi^{2}}{\tau^{3}}
$$

We use Eqs. (144) and (145) to write

$$
\begin{aligned}
& \frac{\partial x}{\partial \tau} \frac{\partial x}{\partial \phi}+\frac{\partial y}{\partial \tau} \frac{\partial y}{\partial \phi}=\left(\frac{\partial R}{\partial \tau}\right)\left(\frac{\partial R}{\partial \phi}\right) \\
& \frac{\partial x}{\partial \tau} \frac{\partial x}{\partial \psi}+\frac{\partial y}{\partial \tau} \frac{\partial y}{\partial \psi}=0
\end{aligned}
$$

Also, from Eq. (154) we get

$$
\begin{aligned}
& \frac{\partial R}{\partial \tau}=-2^{1 / 2} \frac{\phi}{\tau^{2}} \\
& \frac{\partial R}{\partial \phi}=2^{1 / 2} \frac{1}{\tau}
\end{aligned}
$$

and now Eq. (155) allows checking that

$$
\begin{aligned}
& \frac{\partial \theta}{\partial \phi}=-2 \frac{\phi}{\tau^{3}}=\left(\frac{\partial R}{\partial \tau}\right)\left(\frac{\partial R}{\partial \phi}\right) \\
& \frac{\partial \theta}{\partial \psi}=0
\end{aligned}
$$

in explicit agreement with the constraint Eq. (78), as expected.

We notice that $R^{2}=x^{2}+y^{2}$ and this allows getting

$$
\phi^{2}=\frac{1}{2} \tau^{2}\left(x^{2}+y^{2}\right)
$$

and so we can write

$$
\theta(t, x, y)=-\frac{1}{2 t}\left(x^{2}+y^{2}\right)
$$


We also have

$$
g=\frac{2}{\tau^{2}} R^{2}
$$

and now Eq. (88) allows getting

$$
\rho(t, x, y)=\frac{\sqrt{\lambda}|t|}{\sqrt{\left(x^{2}+y^{2}\right)}}
$$

This pair of solutions identifies the circularly symmetric, dilation invariant solutions presented in [1] for the Galileo invariant system in $(2,1)$ dimensions.

We remark that the above solution (154) is a special case of the solution found in [8], which gives another pair of solution to the Galileo invariant system, a pair that presents no dilation invariance anymore. This fact was already known to the author of Ref. [8].

\subsection{Solutions for $d=3$}

Let us now consider the 3 -brane system in $(4,1)$ dimensions. We parametrize this 3-brane with $\left(\phi^{1}, \phi^{2}, \phi^{3}\right)=(\phi, \chi, \psi)$ in space $\mathbf{x}=(x, y, z)$. We choose the spherical Ansatz

$$
\begin{aligned}
& x=R(\tau, \phi) \sqrt{1-\chi^{2}} \cos (\psi) \\
& y=R(\tau, \phi) \sqrt{1-\chi^{2}} \sin (\psi) \\
& z=R(\tau, \phi) \chi
\end{aligned}
$$

In this case we use Eq. (65) to obtain

$$
\begin{aligned}
g_{11} & =\left(\frac{\partial R}{\partial \phi}\right)^{2} \\
g_{22} & =\frac{R^{2}}{1-\chi^{2}} \\
g_{33} & =R^{2}\left(1-\chi^{2}\right)
\end{aligned}
$$

with $g_{i j}=0$ for $i \neq j$. This implies that

$$
g=R^{4}\left(\frac{\partial R}{\partial \phi}\right)^{2}
$$

We then get $g g^{i j}=0$ for $i \neq j$, and for the diagonal elements we have

$$
\begin{aligned}
g g^{11} & =R^{4} \\
g g^{22} & =\left(1-\chi^{2}\right) R^{2}\left(\frac{\partial R}{\partial \phi}\right)^{2} \\
g g^{33} & =\frac{R^{2}}{1-\chi^{2}}\left(\frac{\partial R}{\partial \phi}\right)^{2}
\end{aligned}
$$


We use Eq. (83) to obtain from the equations for $x, y$, and $z$ the same equation for $R=R(\tau, \phi)$

$$
\frac{\partial^{2} R}{\partial \tau^{2}}=R^{4} \frac{\partial^{2} R}{\partial \phi^{2}}+2 R^{3}\left(\frac{\partial R}{\partial \phi}\right)^{2}
$$

This equation for $R(\tau, \phi)$ can also be solved by separating variables. We see that

$$
R(\tau, \phi)=3^{1 / 4}\left(\frac{\phi}{\tau}\right)^{1 / 2}
$$

explicitly solves Eq. (176). This result can be used in Eqs. (78) and (84) to give

$$
\theta(\tau, \phi)=-\frac{1}{4} 3^{1 / 2} \frac{\phi}{\tau^{2}}
$$

We use the spherical Ansatz to write

$$
\begin{aligned}
& \frac{\partial x}{\partial \tau} \frac{\partial x}{\partial \phi}+\frac{\partial y}{\partial \tau} \frac{\partial y}{\partial \phi}+\frac{\partial z}{\partial \tau} \frac{\partial z}{\partial \phi}=\left(\frac{\partial R}{\partial \tau}\right)\left(\frac{\partial R}{\partial \phi}\right) \\
& \frac{\partial x}{\partial \tau} \frac{\partial x}{\partial \psi}+\frac{\partial y}{\partial \tau} \frac{\partial y}{\partial \psi}+\frac{\partial z}{\partial \tau} \frac{\partial z}{\partial \psi}=0 \\
& \frac{\partial x}{\partial \tau} \frac{\partial x}{\partial \chi}+\frac{\partial y}{\partial \tau} \frac{\partial y}{\partial \chi}+\frac{\partial z}{\partial \tau} \frac{\partial z}{\partial \chi}=0
\end{aligned}
$$

Also, from Eq. (177) we get

$$
\begin{aligned}
& \frac{\partial R}{\partial \tau}=-\frac{1}{2} 3^{1 / 4}\left(\frac{\tau}{\phi}\right)^{1 / 2} \frac{\phi}{\tau^{2}} \\
& \frac{\partial R}{\partial \phi}=\frac{1}{2} 3^{1 / 4}\left(\frac{\tau}{\phi}\right)^{1 / 2} \frac{1}{\tau}
\end{aligned}
$$

We use Eq. (178) to see that

$$
\frac{\partial \theta}{\partial \phi}=-\frac{1}{4} 3^{1 / 2} \frac{1}{\tau^{2}}
$$

which shows explicit agreement with the constraint Eq. (78).

To obtain $\theta$ in terms of the variables $(t, x, y, z)$ we notice that the spherical Ansatz allows writing

$$
x^{2}+y^{2}+z^{2}=R^{2}=3^{1 / 2} \frac{\phi}{\tau}
$$

We use this result in Eq. (178) to obtain

$$
\theta(t, x, y, z)=-\frac{1}{4 t}\left(x^{2}+y^{2}+z^{2}\right)
$$


The density is obtained with Eqs. (88) and (172). It reads

$$
\rho(t, x, y, z)=2 \sqrt{\frac{2}{3}} \lambda \frac{|t|}{\sqrt{x^{2}+y^{2}+z^{2}}}
$$

and together with Eq. (186) forms a pair of solutions of the Galileo invariant system (11) in $(3,1)$ dimensions. This pair of solutions is exactly the dilation invariant solutions found in [1] for the Galileo invariant system in $d=3$, with the density-dependent interaction $V(\rho)=\lambda / \rho$. They are the solutions (57) and (58) in the case $d=3$.

\section{Generalization}

The results obtained in Sec. 3 for the $d$-brane system in $d=2$ and 3 can be naturally extended to dimensions higher than $d=3$. However, instead of generalizing the former spherical Ansatz to arbitrary dimension we follow another route, which concerns generalizing the results we have already obtained in the former Sec. 3. We do this by first writing Eqs. (153) and (176) together

$$
\begin{array}{ll}
\frac{\partial^{2} R}{\partial \tau^{2}}=R^{2} \frac{\partial^{2} R}{\partial \phi^{2}}+R\left(\frac{\partial R}{\partial \phi}\right)^{2} & (d=2) \\
\frac{\partial^{2} R}{\partial \tau^{2}}=R^{4} \frac{\partial^{2} R}{\partial \phi^{2}}+2 R^{3}\left(\frac{\partial R}{\partial \phi}\right)^{2} & (d=3)
\end{array}
$$

These results suggest the general behavior

$$
\frac{\partial^{2} R}{\partial \tau^{2}}=R^{2(d-1)} \frac{\partial^{2} R}{\partial \phi^{2}}+(d-1) R^{2(d-1)-1}\left(\frac{\partial R}{\partial \phi}\right)^{2}
$$

This equation is nonlinear in all but the $d=1$ case, where it reproduces the wave equation already considered in the former Sec. 3 .

Fortunately, we can solve Eq. (190) explicitly in the general $d>1$ case. We have the solution

$$
R(\tau, \phi)=\left(d^{1 / 2} \frac{\phi}{\tau}\right)^{1 /(d-1)}
$$

On the other hand, we also have the results

$$
\begin{array}{ll}
g=R^{2}\left(\frac{\partial R}{\partial \phi}\right)^{2} & (d=2) \\
g=R^{4}\left(\frac{\partial R}{\partial \phi}\right)^{2} & (d=3)
\end{array}
$$


They suggest the general behavior

$$
g=R^{2(d-1)}\left(\frac{\partial R}{\partial \phi}\right)^{2}
$$

The results for $\theta$ for $d=2$ and 3 are given by Eqs. (155) and (178). They can be rewritten as

$$
\begin{aligned}
& \theta(\tau, \phi)=-\frac{\phi^{2}}{\tau^{3}}=-\frac{1}{2} \frac{1}{\tau}\left(2^{1 / 2} \frac{\phi}{\tau}\right)^{2} \quad(d=2) \\
& \theta(\tau, \phi)=-\frac{1}{4} 3^{1 / 2} \frac{\phi}{\tau^{2}}=-\frac{1}{2} \frac{1}{2 \tau}\left[3^{1 / 4}\left(\frac{\phi}{\tau}\right)^{1 / 2}\right]^{2} \quad(d=3)
\end{aligned}
$$

They and the result given by Eq. (191) for $R(\tau, \phi)$ allow the following generalization

$$
\theta(\tau, \phi)=-\frac{1}{2} \frac{R^{2}}{(d-1) \tau}
$$

which is valid under the restriction $d>1$. For the density we use Eqs. (88) and (194) to get

$$
\rho(\tau, \phi)=\sqrt{\frac{2 \lambda}{d}}(d-1) \frac{|\tau|}{R}
$$

These results can be rewritten as

$$
\begin{array}{r}
\theta(t, \mathbf{x})=-\frac{1}{2} \frac{r^{2}}{(d-1) t} \\
\rho(t, \mathbf{x})=\sqrt{\frac{2 \lambda}{d}}(d-1) \frac{|t|}{r}
\end{array}
$$

after changing $(\tau, \phi) \rightarrow(t, \mathbf{x})$ and using $r=\sqrt{\mathbf{x} \cdot \mathbf{x}}$. They are the results (57) and (58), found in Ref. [1] for the Galileo invariant system (1]) in $(d, 1)$ dimensions, for $d>1$.

There are at least two other interesting issues related to Eq. (190). The first one is that it can be obtained from the Lagrangian density

$$
\mathcal{L}=\frac{1}{2}\left(\frac{\partial R}{\partial \tau}\right)^{2}-\frac{1}{2} R^{2(d-1)}\left(\frac{\partial R}{\partial \phi}\right)^{2}
$$

which can be rewritten in the form

$$
\mathcal{L}=\frac{1}{2} \mathcal{G}^{\alpha \beta} \partial_{\alpha} R \partial_{\beta} R
$$

where we are using that

$$
\partial_{\alpha}=\left(\frac{\partial}{\partial \tau}, \frac{\partial}{\partial \phi}\right)
$$


and

$$
\begin{aligned}
\mathcal{G}^{00} & =1 \\
\mathcal{G}^{01} & =\mathcal{G}^{10}=0 \\
\mathcal{G}^{11} & =-R^{2(d-1)}
\end{aligned}
$$

in the effective $(1,1)$ dimensional space-time $(\tau, \phi)$. Here we introduce the determinant $\mathcal{G}=\operatorname{det}\left(\mathcal{G}^{\alpha \beta}\right)=-R^{2(d-1)}$. In general it depends on the point $(\tau, \phi)$, but in the $d=1$ case it becomes constant, independent of both the $\phi$ and $\tau$ coordinates of the bidimensional space-time.

The second issue related to Eq. (190) concerns the case of considering $\tau$-independent spherical Ansatz, that is, of reducing $R(\tau, \phi)$ to $R(\phi)$. In this case Eq. (190) becomes

$$
R \frac{d^{2} R}{d \phi^{2}}+(d-1)\left(\frac{d R}{d \phi}\right)^{2}=0
$$

A direct consequence of $R$ being $\tau$-independent is that

$$
\frac{\partial g}{\partial \tau}=0
$$

Furthermore, from Eqs. (194) and (207) we obtain

$$
\frac{\partial g}{\partial \phi}=0
$$

and so $g$ is constant. From Eq. (84) we then get

$$
\theta=\frac{1}{2} g \tau
$$

which is independent of $\phi$ and so compatible with the constraint Eq. (78). An explicit illustration is given after solving Eq. (207). Here we see that

$$
R(\phi)=\phi^{1 / d}
$$

solves Eq. (207). Also we use Eq. (194) to get

$$
g=\frac{1}{d^{2}}
$$

This result and Eq. (210) allow writting

$$
\theta=\frac{1}{2 d^{2}} \tau
$$


Eqs. (211) and (213) give another solution for the $d$-brane in $d>1$ spatial dimensions. We remark that solutions to the above second-order Eq. (207) can also be obtained via the following first-order equation

$$
\frac{d R}{d \phi}=\frac{1}{d} R^{1-d}
$$

We notice that the above solution (211) solves the first-order Eq. (214). We postpone to a future work further investigations on this and on other related issues.

\section{Comments and conclusions}

In this paper we have found solutions for the relativistic $d$-brane system in $(d+1,1)$ dimensions for $d=1$ and for $d>1$. The $d$-brane system presents spherically symmetric solutions in $d=2,3$ that are directly related to the spherically symmetric solutions introduced in [1] for the Galileo invariant system in $(2,1)$ and in $(3,1)$ dimensions. These results were generalized to higher spatial dimensions, and so we obtained solutions of the relativistic $d$-brane system in $(d+1,1)$ dimensions that reproduce the solutions (57) and (58) of the Galileo invariant system (1) in $(d, 1)$ dimensions, for $d>1$.

These solutions are very different from the solutions one finds in $(1,1)$ dimensions. In $d=1$ the Galileo invariant system was solved exactly. The general behavior follows as in [2], which solved the equations of motion following the route of linearization of mechanics of fluids [3]. In Ref. [2] one also finds infinity sets of conserved quantities, which are proper to systems engendering general integrability. The behavior of general integrability in $d=1$ shows up in the present investigations via the presence of the wave equation, which is linear and is solved exactly by standard method. The 1-brane route to solutions to the Galileo invariant system in $(1,1)$ dimensions is very interesting since it allows obtaining the static solutions and identifying how the BPS solutions of that dynamical system appear in the 1-brane system.

The investigations done in the present paper introduce further issues, for instance the problem of searching for other solutions. In connection with Ref. [1] one can ask about the possibility of transforming solutions to new ones, using the field-dependent coordinate transformations generated by $\mathbf{G}$ in Eq. (21). In the hydrodynamical formulation of quantum mechanics we can ask about the possibility of not only introducing diffusion 18 but also going beyond the description of pure states [15]. Other interesting investigations 23] have been done, generalizing not only the way the fluid mechanical system interacts but also how the kinematical contribution may modify its dynamical behavior.

We can also reformulate the light-cone description of the $d$-brane in the way that leads to the Born-Infeld equation [24], as presented in Refs. [7, 8], for instance. As one knows, the Born-Infeld equation arises after a nonlinear modification of standard electrodynamics, and presents interesting properties [25] and connections to fluid mechanics and other issues, as the ones investigated recently in Refs. [26, 27]. Another interesting issue concerns 
the fact that $d$-branes are extended objects that can be related to matrix theory and as such may be of interest for instance in the context of holomorphic configurations recently considered in Ref. [28].

\section{Acknowledgments}

The author would like to thank Roman Jackiw, from whom he has learned quite a few interesting things related to the subject of this work. He also thanks Jens Hoppe and Washington Taylor for interesting comments and discussions. He is grateful to the Center for Theoretical Physics, MIT, for hospitality.

\section{References}

[1] D. Bazeia and R. Jackiw, Ann. Phys. (NY), in press, hep-th/9803165.

[2] R. Jackiw and A.P. Polychronakos, Faddeev Festschrift Proceedings, hep-th/9809123.

[3] L. Landau and E. Lifschitz, Fluid Mechanics, 2nd ed. (Pergamon, Oxford UK, 1987).

[4] E. Madelung, Z. Phys. 40, 322 (1926).

[5] E. Merzbacher, Quantum Mechanics, 3rd ed. (Wiley, New York, 1998).

[6] J. Hoppe, MIT PhD Thesis (unpublished, 1982).

[7] M. Bordemann and J. Hoppe, Phys. Lett. B 317, 315 (1993).

[8] J. Hoppe, Phys. Lett. B 329, 10 (1994).

[9] J. Hoppe, preprint, hep-th/9503069.

[10] A. Jevicki, Phys. Rev. D 57, R5955 (1998).

[11] A.M.J. Schakel, Mod. Phys. Lett. B 10, 999 (1996).

[12] N. Ogawa, preprint, hep-th/9801115.

[13] A.M.J. Schakel, preprint, cond-mat/9805152.

[14] L.D. Faddeev and R. Jackiw, Phys. Rev. Lett. 60, 1692 (1988).

[15] U. Fano, Rev. Mod. Phys. 29, 74 (1957).

[16] E. Wigner, Phys. Rev. 40, 749 (1932). 
[17] P. Carruthers and F. Zachariasen, Rev. Mod. Phys. 55, 245 (1983).

[18] H.D. Doebner and G.A. Goldin, J. Phys. A 27, 1771 (1994).

[19] L. Susskind, Phys. Rev. 165, 1535 (1968).

[20] C. Duval and P.A. Horvathy, unpublished.

[21] J. Hoppe, private communication.

[22] M.K. Prasad and C.M. Sommerfield, Phys. Rev. Lett. 35, 760 (1975); E.B. Bogomol'nyi, Sov. J. Nucl. Phys. 24, 449 (1976).

[23] R. Jackiw and A.P. Polychronakos, preprint, hep-th/9902024.

[24] M. Born and L. Infeld, Proc. R. Soc. London A 144, 425 (1934).

[25] M. Arik, F. Neyzi, Y. Nutku, P.J. Olver, and J.M. Verosky, J. Math Phys. 30, 1338 (1989).

[26] C.G. Callan and J.M. Maldacena, Nucl. Phys. B 513, 198 (1998).

[27] G.W. Gibbons, Nucl. Phys. B 514, 603 (1998).

[28] L. Cornalba and W. Taylor, Nucl. Phys. B 536, 513 (1998). 